\title{
Activation of innate immunity by a probiotic bacterium and a fermented milk containing this micro-organism
}

\author{
C. Maldonado Galdeano ${ }^{1,2}$, A. de Moreno de Leblanc ${ }^{1}$, C. Dogi ${ }^{1}$, S. Chaves $^{1}$, E. Carmuega ${ }^{3}$, \\ R. Weill ${ }^{4}$ and G. Perdigón ${ }^{1,2}$ \\ ${ }^{1}$ CERELA, Chacabuco 145, 4000 Tucumá, Argentina, ${ }^{2}$ Cát Inmunología, Fac Bqca, Qca y Fcia, Universidad Nacional \\ de Tucumán, Argentina, ${ }^{3}$ Nutritia, Buenos Aires, Argentina and ${ }^{4}$ Dto Investigación y Desarrollo, Danone SA, Argentina
}

\begin{abstract}
Improvement of the immune status of the host is one of the beneficial properties attributed to probiotics. Previous results have shown that the effect induced by a probiotic strain Lactobacillus casei CRL 431 is through innate immunity ${ }^{(1,2,3)}$. The present work investigated the behaviour of another probiotic bacterium Lactobacillus casei DN 114001 in relation to the interaction between the epithelial and immune cells, using mice as the experimental model. Using electron microscopy and fluorescent bacteria it was demonstrated that this bacterium interacts with the intestinal epithelial cells and the small fragments of the bacterium can internalize and make contact with the immune cells (macrophages and dendritic cells) present in Peyer patches and in the lamina propria of the small and large intestine. These antigenic particles remain in the gut for $72 \mathrm{~h}$, similar to other particulate antigens. A subsequent study investigated the effect of the inclusion of this probiotic bacterium in fermented milk on the mucosal innate immune system of the gut in BALBc mice. The fermented milk was administered to the mice for five consecutive days, which was previously determined to be the optimal dose to stimulate the immune response. At the end of the administration period the animals were killed and the small and large intestine was removed for the determination of: (a) the number of IgA- and cytokine (IL-10, interferon $\gamma$ (IFN- $\gamma$ ), TNF $\alpha$ )-producing cells in histological slices of both intestines; (b) the expression in the lamina propria of the small intestine of the different receptors present in the immune cells involved in the innate immunity, e.g. mannose receptor CD206 present in macrophages or on the dendritic cell surface (this receptor participates mainly in the internalization process and in antigen clearance); (c) Toll-like receptor 4 (TLR4), which is involved in the adhesion and proinflammatory signals induced by pathogenic bacteria, as a measure of possible adverse effects. Increases were found in the number of IgA-, TNF $\alpha$ - and IFN $\gamma$-producing cells in both intestines. The anti-inflammatory cytokine IL-10 also showed a significant increase in relation to the control (without fermented milk). This increase may have been induced to down regulate the mucosal response. The receptor CD206 was slightly increased, but TLR4 was unchanged. These results showed that innate immunity is involved in the gut mucosal immune activation observed, and led to an investigation of the effect of the consumption of this fermented milk in early period of the life, when the maturation of the innate immune cells occurs. After weaning, newborn mice received the fermented milk until the adulthood ( $45 \mathrm{~d}$ of age). The number of cells positive for maturation markers, such as F4/80+ cells (macrophages) and 33D1 + cells (dendritic cells) were determined in the small intestine. The expression of these markers increased in treated animals compared with the control group (newborn mice not receiving fermented milk).

These results demonstrate that the probiotic bacterium or its fragments interact with epithelial and phagocytic immune cells associated with the gut. This observation is reflected in the results obtained with the fermented milk, which show an increase in the number of CD206 receptors and activation of the immune cells associated with the gut, with an increase in cytokine- and IgA+-producing cells. The results for the specific markers of maturation of the macrophages and dendritic cells in adult mice (45 d of age) that received the fermented milk after weaning are in agreement with those of previous studies.

It has been demonstrated that fermented milk containing the probiotic strain L. casei DN 114001 can modulated the gut immune response mainly through the innate immune system by increasing the receptors related to maturation of the macrophages and dendritic cells associated with the gut, by activating these cells and by increasing the number of $\operatorname{IgA}+\mathrm{B}$-cells.
\end{abstract}

1. Galdeano CM \& Perdigón G (2004) J Appl Microbiol 97, 673-681.

2. Maldonado Galdeano C, de Moreno de Leblanc A, Vinderola G, Bibas Bonet ME \& Perdigón G (2007) Clin Vaccine Immunol 14, 485-492.

3. Maldonado Galdeano C \& Perdigón G (2006) Clin Vaccine Immunol 13, 219-226. 